Tillman ${ }^{1}$ and also Fermi ${ }^{2}$ have shown that the activity of silver increases to about 10-30 per cent when paraffin wax is cooled through the same range of temperatures as in our experiments. The only difference in the experimental conditions consisted in the fact that Tillman and Moon used a layer of cooled paraffin wax $1.7 \mathrm{~cm}$. thick while in our experiments the thickness of this layer was about $10 \mathrm{~cm}$. The discrepancy of the results may be explained by the assumption that the process of slowing down neutrons is accompanied by the absorption. In fact, if cooled paraffin wax absorbs slow neutrons more strongly than the paraffin at room temperature, then the decrease of the number of neutrons reaching the target in the case of a thick layer of cooled paraffin wax may counterbalance the increase of activity of neutrons.

We repeated our experiments under the former conditions and also measured the temperature effect using different thicknesses of paraffin eylinders containing the silver target. Neutrons before reaching these cylinders were strongly retarded. Experiments were made with three cylinders, $0.8 \mathrm{~cm}$., $1 \mathrm{~cm}$. and $1.7 \mathrm{~cm}$. thick respectively. In all three cases, in cooled paraffin wax an increase of activity of silver was observed which diminished with the increase of thickness of paraffin. The inereases amounted to 45, 24 and 13 per cent respectively. There was no increase of activity when the thickness of the paraffin layer was about $10 \mathrm{~cm}$. The range of thicknesses investigated was greater than the mean free path of slow neutrons $(0.5 \mathrm{~cm}$.). At smaller thicknesses, a decrease of the influence of temperature should be again observed.

Our experiments prove the existence of the tem. perature effect and demonstrate the absorption of slow neutrons by paraffin. The absorption becomes stronger as the paraffin is cooled, that is, increases with the decrease of velocities of thermal neutrons.

The effective cross-section of neutrons calculated from our data, assuming the absorption by protons in paraffin wax, is about $10^{-24} \mathrm{~cm}$. sq. The existence of the absorption of slow neutrons explains very well the fall of activity with great retarding thicknesses of water in the well-known experiments of Westcott and Bjerge ${ }^{3}$. The results of Lea's ${ }^{4}$ experiments also may be explained in the same way, if under the conditions of his experiments the slow neutrons were present in sufficient number.

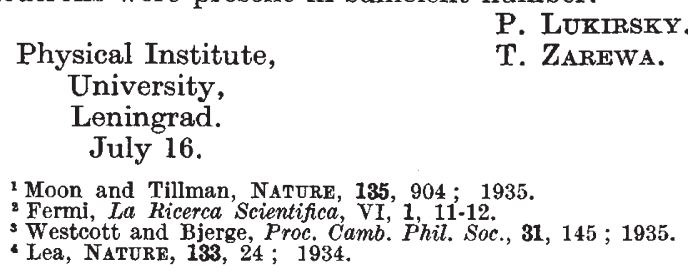

\section{Suggested Improvements of Morse's Rule}

MorsE's empirical relation ${ }^{1}$ between $r_{e}$ and $\omega_{e}$ for di-atoms has recently formed the subject of considerable discussion. It has been amended by three authors, $(A)$ Allen and Longair ${ }^{2}$, who introduce the 'reduced mass' $\mu,(B)$ Badger', who utilises the bondconstant $k_{0}$ derived from a pendulum-type formula, and $(C)$ one of us (C. H. D. C. $)^{4}$, who uses the group number $n$. It is noteworthy that each of these modifications introduces period constants, varying discontinuously between different molecular periods.
The amendments assume interest in view of the preliminary theoretical attack on the problem from the point of view of wave-mechanics by Newing ${ }^{5}$, whose general conclusion is : "We shall expect a law of the type that has been suggested by Badger and by Clark". Further analysis is promised.

The modifications may be tested in their capacity to reproduce experimental values of $r_{e}$ (in A.) from known $\omega_{e}\left(\right.$ in $\left._{\mathrm{cm}}{ }^{-1}\right)$. The following average ( \pm per cent) errors are found for 31 electronic levels of di-atoms of the $K K$ period : $(A) 2 \cdot 7,(B) 1 \cdot 7,(C) 1 \cdot 2$, as against the unmodified formula: $5 \cdot 3$. The $C$ formula $\left(\omega_{e} r_{e}{ }^{3} \sqrt{ } n \doteq 9.70 \times 10^{-21} \mathrm{~cm}^{2}\right)$ is therefore the best for this period.

Allen and Longair ${ }^{2}$ have stated that the $C$ formula will be inapplicable to isotopic molecules, where the $r_{e}$ 's may be closely alike whilst the $\omega_{e}$ 's are different. The only case cited in the $K K$ period is of $\mathrm{B}^{10} \mathrm{O}$ and $\mathrm{B}^{11} \mathrm{O}$, where in the ground states the $C$ formula gives errors of $-1 \cdot 1$ and $-0 \cdot 2$ per cent, respectively. Since both these numbers are within the average error $( \pm 1 \cdot 2$ per cent), it would appear that in practice the objection of Allen and Longair has no very serious weight.

Further details of the above and kindred calcula. tions will be published elsewhere.

$$
\begin{aligned}
& \text { C. H. Douglas Clark. } \\
& \text { J. L. Stoves. }
\end{aligned}
$$

Department of Inorganic Chemistry, University of Leeds.

$$
\text { Oct. } 9 .
$$

${ }^{1}$ P. M. Morse, Phy8. Rev., (ii) 34, 57 ; 1929.

${ }^{2}$ H. S. Allen and A. L. L̈ongair, Phil. Mag, (vii) 19, 1032,1935 ${ }^{3}$ R. M. Badger, J. Chem. Phys., 2, 128 ; 1934 ; Phys. Rev., (ii) 48 $284 ; 1935$.

133,$873 ; 1934$

833, 873; 1934.

\section{Viscosity of Air and the Electronic Charge}

THE greatest uncertainty in determining the electronic charge $e$ by the oil drop method of Millikan is introduced by the uncertainty in the assumed value of the coefficient of viscosity of air, $\eta$. The value adopted by Millikan in 1917

$$
\eta_{23^{\circ}}=(1822 \cdot 6 \pm 1 \cdot 2) \times 10^{-7}
$$

is probably too low, and its accuracy overestimated, as is pointed out by Shiba ${ }^{1}$.

Considering the fundamental importance of the constant $e$, I have undertaken a new determination of $\eta$, using the rotating cylinder method also em. ployed by Millikan and his co-workers ${ }^{2,3}$ : An inner cylinder of electron metal, suspended vertically by a fine phosphor-bronze wire between two guard cylinders of equal diameter is deviated from its equilibrium position through an angle $\varphi$ by a concentric outer cylinder, rotating with constant velocity, $\eta$ being calculated from the equation

$$
\eta=\frac{I\left(b^{2}-a^{2}\right) \cdot \varphi t}{2 a^{2} b^{2} l T^{2}}, \text { where }
$$

$a=$ the radius of the inner cylinder $=2 \cdot 81767 \mathrm{~cm}$. at $20^{\circ}$;

$b=$ the radius of the outer cylinder $=3 \cdot 26628 \mathrm{~cm}$. at $20^{\circ}$ or $=3 \cdot 18328 \mathrm{~cm}$. at $20^{\circ}$ (two different cylinders);

$l=$ the length of the inner cylinder $=9.9981 \mathrm{~cm}$. at $20^{\circ}$;

$t=$ the time of revolution of the outer cylinder $(20-150$ sec. $)$;

$T=$ the period of oscillation of the suspended system (53-128 sec., using different suspensions) ; 